\title{
El modelo de enseñanza personalizada (MEP) como promotor de hábitos saludables
}

\author{
Silvia ARRIBAS-GALARRAGA \\ Izaskun LUÍS-DE COS
}

Datos de contacto:

Silvia Arribas-Galarraga

RESUMEN

Universidad del País Vasco (UPV/EHU)

silvia.arribas@ehu.eus

En el actual paradigma educativo universitario basado en la adquisición de competencias, los grados que forman a futuros docentes han visto la necesidad de actualizar sus metodologías para adaptarse a la demanda de formación. La finalidad de este trabajo consistió en llevar a cabo y evaluar una intervención a través del Modelo de Enseñanza Personalizada (MEP), y en comprobar si su aplicación aumentaba la práctica de actividad físico-deportiva y si se producía un cambio de actitud e intención en esa práctica. Los resultados obtenidos muestran una valoración positiva y un alto grado de satisfacción con el proceso llevado acabo, subrayando la importancia de la personalización del método, que respeta la individualidad, el ritmo de trabajo y la libertad con la que se podía desarrollar el plan. Este trabajo constata la eficacia del Modelo de Enseñanza Personalizada como promotor válido para el incremento de la práctica de actividad físico-deportiva en jóvenes estudiantes.

PALABRAS CLAVE: Hábitos físico-deportivos; Estilo de vida; Condición física; Deporte. 


\title{
The personalized teaching model (MEP) as a promoter of healthy habits
}

\begin{abstract}
In the current university education paradigm based on the acquisition of competences, the degrees that teach future teachers have seen the need to update their methodologies in order to adapt to the education demand. The purpose of this work was to carry out and evaluate a proposal for intervention through the Personalized Teaching Model. And to check if its application increased the practice of physical-sports activity and if there was a change of attitude and intention in that practice. The results showed a positive assessment and a high degree of satisfaction with the process carried out, underlining the importance of the personalization of the method, which respects the individuality, the pace of work and the freedom to develop the plan. This work confirms the effectiveness of the Personalized Teaching Model as a precursor, valid for increasing the practice of physical-sports activity in young students.
\end{abstract}

KEYWORDS: Sport and physical practices habits; Physical condition; Healthy lifestyle.

\section{Introducción}

A raíz de los cambios tanto a nivel curricular como metodológico, acaecidos tras la adecuación al Espacio Europeo de Educación Superior (EEES), el profesorado universitario se ha visto ante la necesidad de orientar su práctica hacia metodologias en las que el estudiante debe saber hacer y aprender a aprender, son metodologias centradas en la adquisición de competencias que dotan al alumnado de herramientas que puedan favorecer su desempeño profesional. (Arribas-Galarraga, Luis- de Cos, Luisde Cos y Urrutia-Gutierrez, 2019).

En la actualidad, en las programaciones de educación física, los modelos de enseñanza están cobrando cada vez mayor presencia. Estos modelos plantean una concepción amplia que engloba tareas, procedimientos, técnicas y estrategias de enseñanza con el objetivo de servir al profesorado en su labor docente (Peiró y Julian, 2015).

En esta nueva concepción del proceso de enseñanza-aprendizaje, el modelo de enseñanza personalizada (MEP) destaca por su contribución al paradigma basado en la adquisición de competencias que se caracteriza porque el alumnado debe tomar la iniciativa en su aprendizaje; "se convierte en un elemento de formación personal en el que la responsabilidad y capacidad de autogestión se dibujan como los pilares fundamentales" (Contreras, Arribas-Galarraga, y Gutiérrez, 2017,p.73). Se le presenta el total de los contenidos a trabajar en un cuadernillo o dossier, y es el estudiante quien, de forma autónoma, progresa a través de la tarea 
previamente diseñada. Cada alumno ha de conseguir un mínimo establecido, pero debe comprometerse a hacer todo lo que pueda por conseguir el mayor nivel posible. El alumnado, bajo la supervisión del profesorado, es protagonista y responsable de su propio aprendizaje. Una de las claves del modelo de enseñanza personalizada es que permite que al alumnado aprendan lo más rápido que puedan o tan despacio como lo necesite, (Metzler 2000; 2005., en Contreras y cols. 2017, p 74).

En esta línea, Romero y Crisol (2012) realizaron una investigación sobre el trabajo autónomo, señalando que este tipo de metodologías se enmarcan a la perfección dentro del paradigma educativo que exige el EEES, permitiendo al alumnado adquirir una serie de competencias que les servirán en su futuro profesional.

El MEP es un modelo innovador, que persigue dotar al alumnado de conocimientos, habilidades y actitudes con el fin de favorecer su implicación en las actividades fisico-deportivas (Peiró y Julian, 2015). En esta metodología el protagonismo recae sobre el o la estudiante, que debe trabajar de una forma activa, individual y crítica para alcanzar los objetivos y tareas propuestas. El docente, por su parte, organiza los contenidos de aprendizaje y las tareas dibujando el objetivo a alcanzar (Calderero, Aguirre, Castellanos, Peris y Perochena, 2014). La principal labor del docente consiste en lograr involucrar al alumnado de forma activa y responsable en su trabajo, respetando el ritmo y estilos de aprendizaje de cada estudiante (Contreras y cols., 2017).

Entre las funciones del profesorado en general y del de educación física en particular, además de adaptarse a los cambios a nivel curricular y metodológico, se encuentra la de hacer frente a las necesidades de la sociedad actual. En una sociedad en la que se está produciendo una rápida transformación en los estilos de vida, uno de los retos a los que se enfrenta este colectivo es el de provocar el gusto por la práctica de actividad físicodeportiva e inculcar hábitos activos tan necesarios en una sociedad en la que el sedentarismo y la obesidad están cada día más presentes.

$\mathrm{El}$ incremento del sedentarismo, a consecuencia, entre otras causas, del ocio tecnológico (Castro-Sánchez, Linares-Manrique, Sanromán Mata, Pérez-Cortés, 2017; Castro, Martínez, Zurita, Chacón, Espejo, y Cabrera, 2015; Díaz y Aladro, 2016), ha llevado a los jóvenes al abandono de la prática físico-deportiva. Numerosos estudios confirman el alto porcentaje de adolescentes y jóvenes que no realizan actividad fisico-deportiva (CSD, 2011; Reverter, Plaza, Jové y Hernández, 2014; Tapia, 2019), así como la disminución de dicha práctica en los jóvenes y jóvenes adultos a medida que aumenta la edad (Beltrán-Carrillo, Devís-Devís y Peiró-Velert, 2012; Isorna, Ruiz y Rial, 2013; Luis de Cos, 2014; Navas y Soriano, 2016; Ramos, Rivera, Moreno y Jiménez-Iglesias, 2012). Como consecuencia, la necesidad de una investigación educativa que genere conocimiento sobre cómo establecer en la adolescencia y juventud un estilo de vida activo y saludable se ha convertido en prioritaria (Hernández y cols., 2008; Tapia, 
2019; Rodríguez-Torres, Páez-Granja, Paguay-Chávez y Rodríguez-Alvear, 2018), para poner a disposición de educadores y profesionales herramientas para fomentar estilos de vida saludables en los jóvenes (Luis de Cos, Luis de Cos y Arribas-Galarraga, 2017).

La principal conclusión de los trabajos revisados hace pensar en la necesidad de concienciar a la juventud de la importancia de la actividad física en su vida cotidiana y dotarles de herramientas para la gestión de su propia práctica físico-deportiva. Por ello, la finalidad de este trabajo consistió en llevar a cabo y evaluar una propuesta de intervención a través del Modelo de Enseñanza Personalizada (MEP), y comprobar si su aplicación aumentaba la práctica de actividad físico-deportiva y si se producía un cambio con respecto a la intención de esa práctica. Los objetivos que se derivan son:

(1) Estudiar la participación y el grado de satisfacción del alumnado con del Modelo de Enseñanza Personalizada y analizar la percepción de la mejora de la condición física a través del mismo.

(2) Comprobar si el alumnado universitario es capaz de incrementar la práctica físico-deportiva a través del MEP.

(3) Analizar el cambio de actitud e intención hacia la práctica físicodeportiva.

\section{Método}

Diseño y metodología

Se trata de un estudio de diseño quasi-experimental, de naturaleza descriptiva. La metodologia utilizada es mixta: cuantitativa para analizar los datos recogidos a través de los cuestionarios y cualitativa para analizar las reflexiones recogidas en el dossier.

Participantes

En el estudio participaron dos grupos de tercer curso del Grado en Educación Primaria; un total de 103 alumnas y alumnos que cursaron la asignatura Educación Física y su Didáctica.

\section{Instrumentos}

En la recogida de datos se utilizó un cuadernillo o dossier, a modo de tutorial, diseñado "ad hoc" y denominado "Plan de Mejora". En el se detallaban las actividades a realizar, los niveles de dificultad a conseguir, etc. además de incorporar los siguientes instrumentos:

- Cuestionario sobre hábitos de práctica físico-deportiva también para recoger información al inicio y al final del proceso (cuestionario 1).

- Test de condición física propuestos para el inicio y final del proceso (condición general: test 1 , resistencia: test 2, y flexibilidad: test 3).

- Reflexiones del alumnado sobre el MEP, que se presenta 
en la parte final del dossier.

- Cuestionario "ad hoc" para recoger información relativa a la metodología desarrollada con el MEP (cuestionario 2), que se pasa al final del proceso.

\section{Procedimiento}

Tras explicar las bases teóricas de MEP se procedió al reparto del dossier (Plan de Mejora) entre el alumnado. Se dio tiempo suficiente para leer el documento y rellenar el cuestionario 1 (hábitos de práctica físicodeportiva). A continuación, se pasaron los test de condición física propuestos en el dossier (test 1, 2 y 3 ).

Durante las siguientes 8 semanas tuvo lugar el desarrollo de la actividad (Plan de Mejora) que se detalla más adelante.

Tras finalizar el periodo previsto, se volvió a pasar la encuesta de hábitos (cuestionario 1), se realizaron los test de condición física (test 1, 2 y 3), y se solicitó al alumnado que cumplimentara el apartado relativo a la reflexión así como el cuestionario relativo a la metodología (cuestionario 2). Finalmente se recogió el dossier.

\section{El Plan de Mejora}

La metodología utilizada (MEP) en el desarrollo del Plan de Mejora, busca convertir el aprendizaje en un elemento de formación personal, en el que la responsabilidad y la capacidad de autogestión son los pilares fundamentales (Contreras y cols., 2017).

El presente estudio se llevó a cabo durante 8 semanas en las que se combinaron sesiones presenciales y no presenciales. El programa se inició con la entrega y lectura del dossier para subsanar posibles dudas. E1 alumnado debía responder al reto propuesto gestionando de forma autónoma las actividades propuestas en dicho documento. A partir de ese momento, se solicitó al alumnado que respondiera el cuestionario 1 (hábitos físico-deportivos), para posteriormente proceder a la pasación inicial de los test 1, 2 y 3 (resistencia, condición física general y flexibilidad), que aportarían datos para verificar la posible mejora de los participantes.
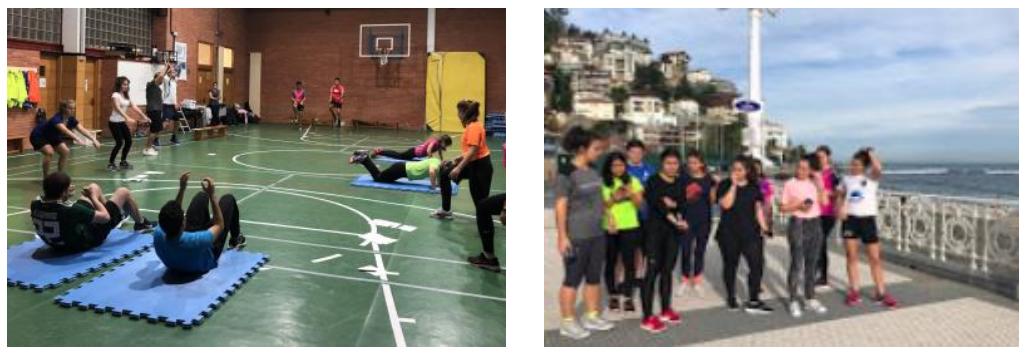


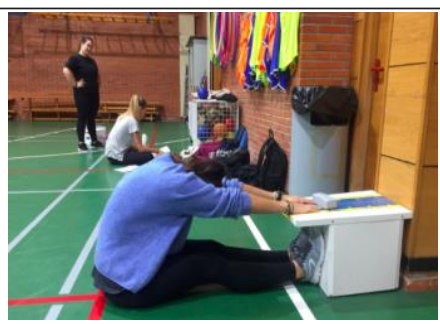

Figura 1. Test de condición general, resistencia y flexibilidad.

Durante las siguientes 8 semanas el alumnado dispuso de una hora semanal presencial para ir desarrollando las tareas presentadas por niveles de dificultad y que deberian de cumplimentar de forma individual en el dossier facilitado por el profesorado. Se animaba a realizar la actividad fisica fuera de las horas lectivas, con el objetivo de promover su mejora fisica y el gusto por realizar ejercicio físico. Se les aconsejaba que realizaran por lo menos una sesión más en su tiempo libre

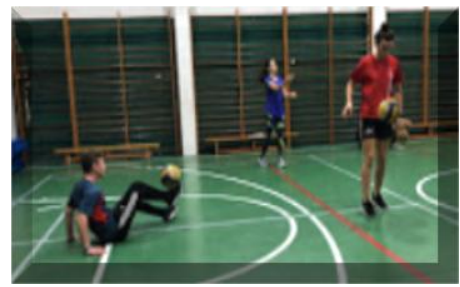

Figura 2. Alumnado trabajando con el MEP de forma autónoma, en sesión presencial.

Para la evaluación del alumnado, se tomaron en cuenta tanto las notas del profesorado, que hacian referencia al proceso del alumnado (capacidad de autogestión, nivel de implicación con la tares, y gestión del tiempo), los niveles alcanzados durante las 8 semanas que duraba el Plan, así como el nivel de mejora de la condición física, para lo que se tomaron en cuenta los test iniciales y finales, ponderados en función del margen de mejora.

Para estudiar el efecto del Plan de Mejora desarrollado a través del Modelo de Enseñanza Personalizada (MEP) cuyos resultados se muestran en este estudio, se tomaron en cuenta las anotaciones del profesorado, los datos obtenidos en el cuestionario 2, así como las reflexiones del alumnado sobre el MEP que se recogen al final del dossier.

Análisis de resultados

Para dar respuesta a los objetivos planteados en el presente proyecto se plantearon análisis cuantitativos: análisis descriptivos y de frecuencias (a través del programa estadístico Statistical Package for the Social Sciencies:SPSS para PC versión 24.0); y análisis cualitativos para interpretar las valoraciones y opiniones recogidas en las declaraciones de 
los y las estudiantes sobre el MEP.

\section{Resultados}

Los resultados que se presentan a continuación se han organizado en función de los objetivos del presente estudio; así, en relación al primer objetivo (Estudiar la participación y el grado de satisfacción del alumnado con el MEP y analizar la percepción de la mejora de la condición física a través del mismo), se observó una alta implicación y participación durante todo el desarrollo del programa. Durante las sesiones presenciales el alumnado mostró interés en la realización de los ejercicios, utilizando el tiempo adecuadamente y realizando las actividades propuestas en el Plan de Mejora de forma autónoma. Cuando aparecían dudas sobre la ejecución de un ejercicio concreto, preguntaban a la profesora quien les instaba a utilizar el soporte detallado en el dossier (principalmente enlaces a tutoriales web). En ocasiones consultaban a sus compañeros y compañeras.

Al preguntar al alumnado sobre estas cuestiones, en una escala donde 0 ere nada/ninguna y 5 era mucho/total, valoraron su participación con una puntuación media de 3.88, su implicación con 3.84 y su interés con un 3.64 puntos promedios. Además, valoraron lo cómodos que se sintieron en la realización del Plan, con una puntuación media de 3.77sobre 5 .

Tabla 1

Puntuaciones Medias sobre Valoraciones en las Sesiones Presenciales (rango 0-5)

\begin{tabular}{lcc}
\hline & $\mathrm{M}$ & $\mathrm{DT}$ \\
& $(\mathrm{n}=103)$ & \\
Interés & 3.64 & 1.34 \\
Participación & 3.88 & 1.33 \\
Implicación & 3.84 & 1.33 \\
Sentirse cómodo & 3.77 & 1.29 \\
\hline
\end{tabular}

El análisis de los datos obtenidos muestra que los participantes declaran estar satisfechas con el trabajo realizado, valorando la satisfacción con la mejora física con un 3.13 puntos promedios y la satisfacción con el aprendizaje con 3.43 (rango 0 - 5). Además, y como respuesta al objetivo 2 (Analizar y reflexionar sobre la percepción de la mejora de la condición física a través del MEP), los resultados indican que el alumnado consideró su mejora de la condición física adecuada (3.03 puntos sobre 5). Además, se constató, a través de los resultados de los test 1,2 y 3 , que todo el alumnado mejoró su condición física general, siendo que la mejora fue mayor en aquellos cuyo rendimiento era menor al iniciarse el programa. 
Otro de los aspectos que se analizó fue además de la satisfacción en su experiencia como alumnos, la utilidad que en su formación como futuros docentes presentaba el MEP. Consideraron el nivel de aprendizaje y de mejora física con 3.41 y 3 puntos promedios respectivamente (rango 0-5), mientras que la satisfacción con el aprendizaje subió a 3,43 puntos y con la mejora fue de 3.16 puntos. Por otra parte, la metodología en su formación y en su faceta personal alcanzan puntuaciones medias de 3.32 y 3.35 respectivamente; asimismo el alumnado considera el MEP adecuado para su formación, (3.39 puntos). Al preguntar de forma global, el 86,8\% valoraron su aprendizaje con 7 puntos o más, en una escala de 0 a 10.

Tabla 2

Puntuaciones Medias sobre Aspectos de Mejora a través del MEP

$\frac{(\text { Rango } 1-5) *(\text { Rango } 1-10)}{\mathrm{M}}$
$(\mathrm{n}=103)$

$3.41 \quad 1.32$

Nivel de aprendizaje

Nivel de mejora fisica

$3.03 \quad 1.34$

Satisfacción con el aprendizaje

$3.43 \quad 1.34$

Satisfacción con la mejora física

$3.16 \quad 1.39$

Adecuación con su formación

$3.39 \quad 1.11$

Utilidad en su formación

Utilidad en el ámbito personal

Puntuación a lo aprendido

7.64 * 1.84

En cuanto a la percepción de mejora de la condición física, prácticamente el $90 \%$ de las opiniones obtenidas declaran ver el MEP muy adecuado para mejorar la condición fisica, además de considerar muy acertada la forma de trabajarlo, por la adecuación al ritmo de cada uno:

".... Te hace dar lo mejor de ti mismo. Encima, este plan es muy recomendable puesto que tienes la suficiente libertad para trabajar...."; (estudiante 99).

"... plan de gran utilidad que he ido siguiendo con gusto y ganas, ya que te da la oportunidad de ir mejorando tus condiciones físicas partiendo de tu nivel y respetando tu ritmo" (estudiante 96).

"El plan de mejora me ha parecido una herramienta muy útil para mejorar mi condición física” (estudiante 75).

En relación al segundo objetivo (Comprobar si el alumnado 
universitario es capaz de incrementar la práctica físico-deportiva a través del MEP y lo ve como modelo promotor de actividad físico-deportiva), algunos de los participantes reconocian que haber puesto en práctica el plan de mejora les habia dado la oportunidad de volver a encontrarse con su yo activo, disfrutar con ello y ver la necesidad de seguir la práctica fisico-deportiva.

"El Plan de Mejora......gracias a ello he vuelto a recuperar la costumbre de ir 2 veces por semana al gimnasio..."(estudiante 39).

"Gracias a este plan mis ganas de practicar actividad físicodeportiva han aumentado y espero seguir entrenando los fines de semana" (estudiante 48).

"...y al ver que he mejorado.....me he quedado muy satisfecha y sé que seguiré practicando ya que he visto que puedo mejorar." (estudiante 69).

Fomentar el trabajo de forma individual y adaptándose a las cualidades de cada individuo es una de las características del modelo de enseñanza personalizado destacado en la mayoría de las opiniones de los participantes, quienes creen que partir de su propio nivel e ir trabajando adecuándose a su ritmo es un acierto para la motivación a seguir trabajando y mejorando.

"La verdad que para que apenas me guste el deporte, me lo he pasado muy bien haciendo el Plan de Mejora, porque me picaba conmigo misma e intentaba superarme" (estudiante 76)

"...el Plan de Mejora me ha encantado, creo que es una forma de trabajar los contenidos de una forma individualizada la cual respeta el ritmo de cada alumno" (estudiante 38).

“...el Plan de Mejora era ajustable a cada persona y que cada uno podia ir mejorando, tuviera el nivel que tuviera” (estudiante 53).

“...dejando a los alumnos realizar las actividades individualmente, todos y cada uno de nosotros hemos mejorado." (estudiante 21)

"Te da la oportunidad de seguir tu ritmo sin presiones y a poder ir avanzando y superando tus propios retos" (estudiante 65).

El ambiente de respeto y confianza que el propio MEP promueve, se ha visto como un aspecto fundamental en el desarrollo de la mejora personal:

"La sensación predominante ha sido la motivación, ya que he visto que me iba superando dia a dia...." (estudiante 59).

"Con el plan de mejora he podido conocer mi cuerpo $y$ he conocido mis limites" (estudiante 84).

"Personalmente,... el Plan de Mejora me ha ayudado para mejorar la imagen que tenia de mi mismo.....Tras realizar las pruebas al principio y al final he podido observar la mejora" (estudiante 87).

Para responder a la segunda parte del objetivo, se preguntó al alumnado si la metodología utilizada era adecuada para impulsar la práctica físico- 
deportiva. El 83\% del alumnado opinó afirmativamente, el 14\% no contestó y el 3\% tuvo dudas. Se han encontrado opiniones muy positivas por parte de los y las participantes respecto a la utilidad de esta metodología como promotora de una vida activa:

"Me parece que es una buena herramienta para adquirir hábitos saludables" (estudiante 3).

"Creo que es una buena forma de promover el deporte y la actividad física y que todos alumnas/os adquieren hábito de hacer ejercicio" (estudiante 8).

"Creo que es una buena manera de motivar a los alumnos a practicar actividad fisico-deportiva y continuar con ella en su tiempo libre" (estudiante10).

"Me parece una buena actividad para que los alumnos realicen ejercicio fuera de clase...." (estudiante 88).

"...para la adquisición de hábitos saludables, me parece que puede ser una buena práctica....” (estudiante 16).

"...es una buena forma de fomentar el ejercicio sobre todo para esa gente que no suele hacerlo." (estudiante 25).

Debido a la experimentación del modelo y a la practicidad de mismo, en algunos casos se ponen en el papel del docente y ven la validez del programa comentando:

"por una parte, con este "plan de Mejora" conseguimos asentar objetivos para la mejora personal de cada alumno. Además, transmitimos la importancia de la actividad físico-deportiva en una rutina diaria. Si esto fuera poco, les ayudamos a obtener unos hábitos saludables $y$ conseguimos inculcarles un afán de superación" (estudiante 78).

El tercer y último objetivo de este estudio proponía analizar el cambio de actitud e intención hacia la práctica físico-deportiva. Para ello se analizó la importancia que los estudiantes de Grado en Educación Primaria otorgaban a la práctica de actividad físico-deportiva. En una escala donde 1 era poco y 9 mucho, la importancia que dieron a su práctica antes de realizar el Plan de Mejora, el alumnado puntuó con una media de 6,80; sin embargo, después del Plan, la puntuación media otorgada subió hasta 7,72 puntos. Observando la figura 2 se puede apreciar como el porcentaje de alumnado que valora su práctica físico-deportiva como muy importante es del $65 \%$ antes de su experiencia, y del $87 \%$ tras realizar el Plan. 


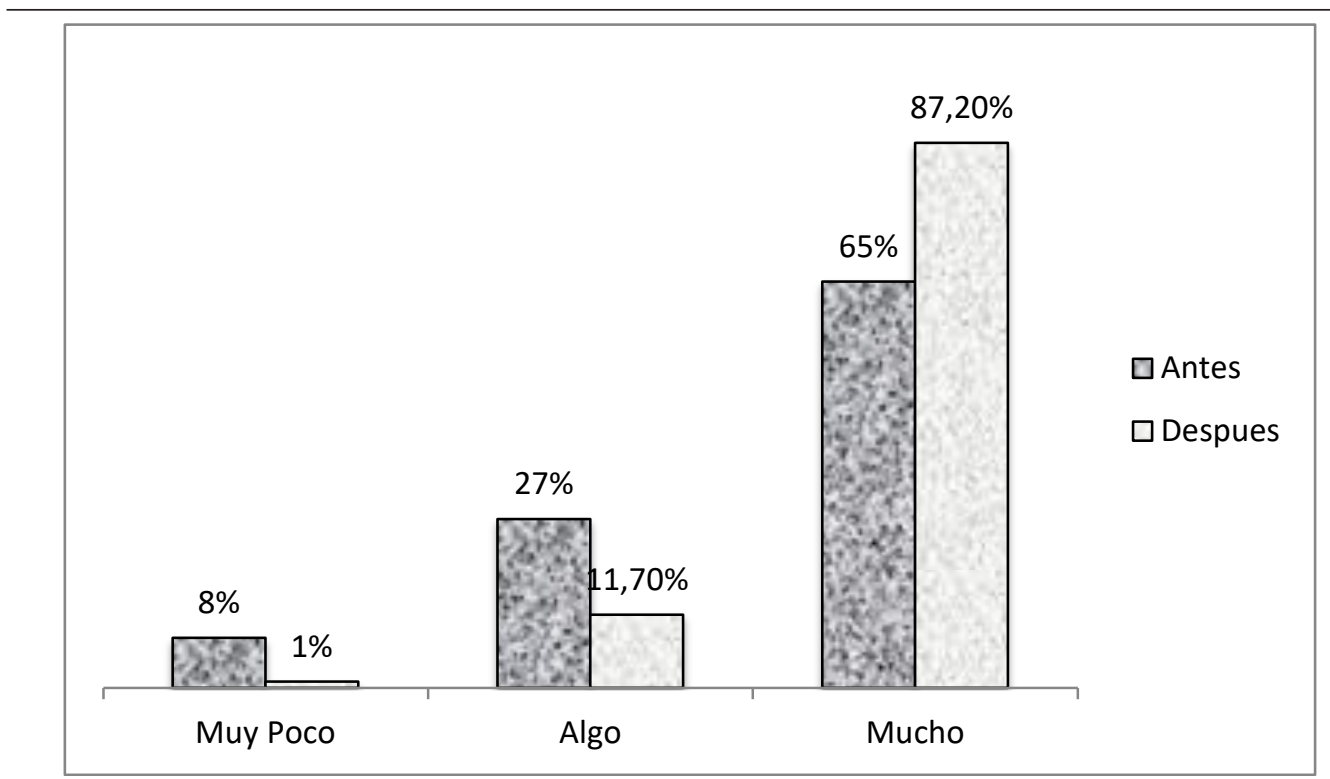

Figura 3. Grado de importancia hacia la Practica físico-deportiva antes y después del Plan de Mejora.

Al preguntar al alumnado sobre su intención de práctica futura, los valores obtenidos señalan que $90,2 \%$ muestra una intención de práctica contundente, el $9,8 \%$ no lo tiene demasiado claro, pero no hay nadie que declare intención de no continuar con su práctica físico-deportiva.

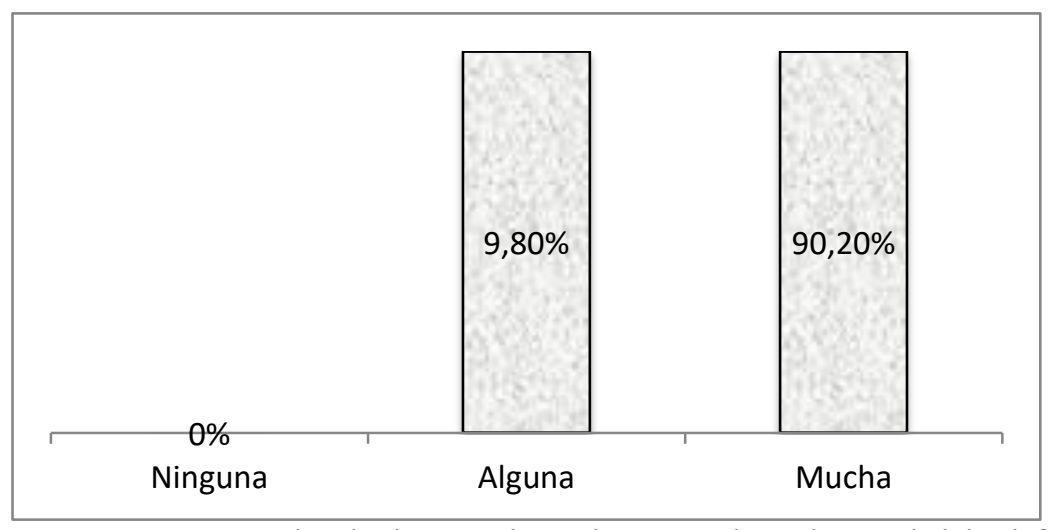

Figura 4. Porcentaje de intención de práctica de actividad físicodeportiva.

A continuación se presentan algunas declaraciones de quienes exponen su intención de seguir practicando:

"Mi intención es seguir con el plan y haciendo ejercicio ya que creo es una buena opción para mejorar la salud" (estudiante 102).

"En mi opinión este "plan de mejora" es una herramienta muy 
adecuada....., también porque en mi caso he sido el punto de partida para una vida más activa (físicamente hablando" (estudiante 21).

"...este plan de mejora me ha venido muy bien para concienciarme del poco ejercicio físico que hacia y para empezar una rutina. He mejorado mucho...Por eso voy a seguir como estos últimos meses haciendo deporte todo lo que pueda" (estudiante 91).

\section{Discusión y conclusiones}

La finalidad del presente estudio consistía en llevar a cabo y evaluar una propuesta de intervención a través del Modelo de Enseñanza Personalizada (MEP), y comprobar si su aplicación aumentaba la práctica de actividad físico-deportiva y si se producía un cambio con respecto a la intención de esa práctica.

Respecto a la evaluación del modelo, en base a los resultados y en virtud de las declaraciones de los protagonistas del Plan de Mejora se ha situado al estudiante en el centro del proceso educativo, hecho que Calderero, y cols. (2014) destacan como característica propia de la educación personalizada. Asímismo se puede afirmar que la puesta en marcha de este programa ha garantizado la adecuación al nuevo paradigma que promueve el sistema universitario, en el cual el alumnado es el protagonista de su propio aprendizaje (Bolivar, 2007; Larasa y Navarro, 2011; Rosa-Guillamón, CarrilloLopez y García-Cantó, 2019). Además, ha permitido a los estudiantes, en la línea de los postulados de Romero y Crisol (2012) desarrollar una serie de competencias que les ayudaran en su futuro profesional.

Los resultados de este estudio muestran que la mayoría de los estudiantes (83\%) consideran que el Modelo de Enseñanza Personalizada puede ser promotor de la práctica de actividad físico-deportiva, destacando la importancia de respetar los ritmos de aprendizaje y el trabajar sin presión. Con este resultado se refuerza la afirmación de Fernandez-Río y cols. (2016) quienes concluyeron que, en los modelos pedagógicos, el alumnado es un elemento esencial en el proceso de enseñanza-aprendizaje, donde adquiere autonomía, responsabilidad y debe entender la práctica de la actividad físicodeportiva como elemento fundamental a lo largo de la vida.

Asimismo, Calderero y cols., (2014), en su propuesta de educación personalizada, aluden a la necesidad de adquirir hábitos operativos buenos. Este hecho queda reforzado a través de este trabajo pues los participantes declaran la adquisición de rutinas de práctica físico-deportivas. En esta misma línea, diversas investigaciones (Calderero, y cols., 2014; Contreras y cols., 2017; Rodriguez, 2006) muestran que el aprendizaje autónomo propone al estudiante como el verdadero protagonista y responsable de su propio proceso de aprendizaje.

En cuanto a la satisfacción con el proceso, se puede afirmar que ha resultado positiva, subrayando la importancia de la personalización del método que respeta la individualidad, el ritmo de trabajo y la libertad con la 
que se podía desarrollar El Plan. Estas características se han considerado parte del éxito del modelo y siguen la idea señalada por Mayorga y Madrid (2010) quienes apuntaron que un modelo basado en el aprendizaje y centrado en el alumnado favorece la implicación, actividad y protagonismo del estudiante. Los participantes en este programa corroboran estos datos, destacando la alta participación, implicación e interés demostrados durante el proceso. Asimismo, los datos obtenidos muestran una alta satisfacción con la mejora física acontecida en el transcurso de la intervención educativa, otorgando parte del éxito de la metodología a la mejora experimentada. Las opiniones obtenidas destacan que la forma de trabajar de manera individual les ha supuesto un reto de superación consigo mismos, y que conseguir una mejora les ha incentivado para seguir trabajando. El alumnado reconoció su propia realidad motriz y puso interés por mejorarla. Apoyándonos en el estudio de Calderero, y cols., (2014) se puede entender que el éxito de la metodología se debe a que se centra en la singularidad de las personas, aumentando la implicación y responsabilidad en el aprendizaje (Rodriguez, 2006). En este sentido se puede deducir que el haber experimentado la mejora en su condición fisica ha ayudado a los participantes a seguir con la propuesta; en cualquier caso, esta propuesta ha promovido que cada persona marcase su propio ritmo de aprendizaje, superando sus propios retos y automotivandose hacía la mejora.

Al hilo de lo anterior, se ha podido observar un cambio de actitud hacía la práctica de actividad físico-deportiva, pues el 90\% del alumnado declara su intención de continuar con su práctica. Además, tras la aplicación de esta modelo de enseñanza se ha observado que las puntuaciones medias otorgadas a la importancia de practicar actividad fisico-deportiva aumentan de modo que el 22\% de los encuestados da más importancia a la práctica físico-deportiva que antes. La aplicación del MEP podría constituir un paso en el largo camino a recorrer para mitigar hábitos sedentarios (CSD, 2011; Reverter, Plaza, Jové y Hernández, 2014; Tapia, 2019), y la disminución en práctica físico-deportiva de jóvenes recogida en diversos estudios (BeltránCarrillo, Devís-Devís y Peiró-Velert, 2012; Isorna, Ruiz y Rial, 2013; Luis de Cos, 2014; Navas y Soriano, 2016; Ramos, Rivera, Moreno y Jiménez-Iglesias, 2012).

Finalmente, el presente estudio conduce hacia una visión esperanzadora en la que la promoción de hábitos de actividad físico-deportiva entre la juventud es una realidad alcanzable. El modelo de enseñanza personalizada que se establece a través de niveles, permite el empoderamiento del alumnado ante su proceso de aprendizaje y otorga un escenario para el desarrollo de la responsabilidad, el conocimiento de las propias fortalezas (y limitaciones) así como para el afrontamiento de desafios personales que genera sensaciones de satisfacción y reto a futuro, en forma de intención de práctica. 


\section{Referencias}

Arribas-Galarraga, S., Luis-de Cos, I., Luis-de Cos, G., Urrutia-Gutierrez, S. (2019). Aprendizaje cooperativo: un proyecto de expresión corporal en el grado de Educación Primaria. Journal of Sport and Health Research. 11(Sup1 1),155-166.

Beltrán-Carrillo, V.J., Devís-Devís, J. y Peiró-Velert, C. (2012). Actividad física y sedentarismo en adolescentes de la Comunidad Valenciana. Revista Internacional de Medicina y Ciencias de la Actividad Física y el Deporte, 12 (45), 122-137.

Bolívar, A. (2007). La planificación por competencias en la reforma de Bolonia de la educación superior: un análisis crítico. Educaçao Temática Digital, 9, 68.

Calderero, J. F., Aguirre, A.M., Castellanos, A., Peris, R.M. y Perochena, P (2014).

Castro, M., Martínez, A., Zurita, F., Chacón, R., Espejo, T. y Cabrera, A. (2015). Uso de videojuegos y su relación con las conductas sedentarias en una población escolar y universitaria. Journal for Educators, Teachers and Trainers, 6(1), 40-51.

Castro-Sánchez, M., Linares-Manrique, M., Sanromán Mata, S., Pérez-

Cortés, A.J. (2017). Análisis de los comportamientos sedentarios, práctica de actividad física y uso de videojuegos en adolescentes. Sportis Sci J, 3 (2), 241-255

Consejo Superior de Deportes (CSD) (2011). Estudio de los hábitos deportivos de la población escolar española. Eds: Consejo Superior de Deportes.

Contreras, O., Arribas-Galarraga, S. y Gutiérrez, D. (2017). Didáctica de la educación física por modelos para educación primaria. Madrid: Sintesis.

Díaz, R. y Aladro, A. (2016). Relación entre uso de las nuevas tecnologías y sobrepeso infantil, como problema de salud pública. RqR Enfermeria Comunitaria, 4(1), 46-51.

Fernandez-Río, J., Calderón, A., Hortigüela-Alcalá, d., Pérez-Pueyo, a. y Aznar-Cebamanos, M. (2016). Modelos pedagógicos en educación física: consideraciones teórico-prácticas para docentes. Revista Española de Educación y Deportes. 413, 55-75.

Hernández, J.L. Velázquez, R.; Martínez $\mathrm{M}^{\mathrm{a}}$ E., Garoz, I., López, C. y López, A. (2008). Frecuencia de actividad física en niños y adolescentes: relación con su percepción de autoeficacia motriz, la práctica de su entorno social y su satisfacción con la Educación Física. Infancia y Aprendizaje 31(1), 79-92.

Isorna, M., Ruiz, F. y Rial, A. (2013). Variables predictoras del abandono de la práctica físico-deportiva en adolescentes. CCD Cultura Ciencia y Deporte, 8, 93-102.

Larasa, I. y Navarro, E. (2011). Hacia el aprendizaje autónomo en la 
universidad elementos para la reflexión y el debate a partir de la experiencia enmarcada en el modelo IKD de la UPV-EHU. III Congreso Internacional "La autogestión del aprendizaje". Girona, Universidad de Girona.

Luis de Cos, I. (2014). Entorno Social y Predicción de la Práctica de Actividad Física y Deporte en Población Adolescente. (Tesis doctoral). Donostia-San Sebastián: Universidad del País Vasco.

Luis-de Cos, I.; Luis-de Cos, G., Arribas-Galarraga, S. (2017). Práctica de actividad física y deporte, asociacionismo e intención de práctica futura en un grupo de adolescentes de 12 a 16 años. Sportis Sci J, 3

419-435.

DOI:https://doi.org/10.17979/sportis.2017.3.3.1899.

Mayorga, M.J. y Madrid, D. (2010). Modelos didácticos y Estrategias de enseñanza en el Espacio Europeo de Educación superior. Tendencias pedagógicas, 15(1), 91-111.

Navas, L. y Soriano, J.A. (2016). Análisis de los motivos para practicar o no actividades físicas extracurriculares y su relación con el autoconcepto físico en estudiantes chilenos. Revista Iberoamericana de psicología del ejercicio y el deporte, 11(1) 69-76.

Metzler, M. (2000). Instructor's manual for the Personalized System Instructions series. Boston, MA: Allyn y Bacon.

Metzler, M. (2005). Instructional models for physical education. Scottsdale, AZ: Holcomb y Hathaway.

Peiró, C. y Julian, J.A. (2015). Los modelos pedagógicos en educación física. Un enfoque más allá de los contenidos curriculares. Tándem Didáctica de la Educación Física, 50, 9-15.

Ramos, P., Rivera, F., Moreno, C. y Jiménez-Iglesias, A. (2012). Análisis de clúster de la actividad física y las conductas sedentarias de los adolescentes españoles, correlación con la salud biopsicosocial. Revista de Psicología del Deporte, 21(1), 99-106.

Rosa-Guillamón, A., Carrillo-López, P.J. y García-Cantó, E. (2019). Learning based on projects. A didactic experience from the physical education area. ESHPA - Education, Sport, Health and Physical Activity. 3(1): 141-159.

Reverter, J., Plaza, D., Jové, M.C. y Hernández, V. (2014). Actividad físicodeportiva extraescolar en alumnos de primaria: el caso de Torrevieja (Alicante). Retos. Nuevas tendencias en Educación Física, Deporte y Recreación 25, 48-52.

Rodríguez, R. (2006). Diseño de entornos para el desarrollo de la autonomía en el aprendizaje. Aula Abierta 87, 89-104.

Rodriguez-Torres, Á. F., Páez-Granja, R. E., Paguay-Chávez, F. W., y Rodríguez-Alvear, J. C. (2018). El profesorado de educación física y 
la promoción de salud en los centros educativos. The Physical Education teaching staff and the health promotion in the educational centers. Arrancada, 18(34), 215-235.

Romero, M.A, y Crisol, E. (2012). Las guías de aprendizaje autónomo como herramienta didáctica de apoyo a la docencia. Aula Abierta, $15,9-3$.

Tapia, A. (2019). Diferencias en los niveles de actividad fisica, grado de adherencia a la dieta mediterránea y autoconcepto físico en adolescentes en función del sexo. Retos. Nuevas tendencias en Educación Física, Deporte y Recreación, 36(36), 185-192. 